\title{
Neonatal exposure to $17 \beta$-estradiol down-regulates the expression of synaptogenesis related genes in selected brain regions of adult female rats
}

\author{
N.S. Radhika ${ }^{\text {a }}$, Vijayakumar Govindaraj ${ }^{\text {b }}$, S.K. Sarangi ${ }^{\text {, }}$, A.J. Rao ${ }^{\text {b,* }}$ \\ a Department of Microbiology and Biotechnology, Bangalore University, Bangalore, India \\ b Department of Biochemistry, Indian Institute of Science, Bangalore, India
}

\section{A R T I C L E I N F O}

\section{Article history:}

Received 12 April 2015

Received in revised form 7 August 2015

Accepted 21 September 2015

Available online 26 September 2015

\section{Keywords:}

Neonatal $17 \beta$-estradiol exposure

Pre-optic area

Synaptogenesis

Hypothalamus

Pituitary

\begin{abstract}
A B S T R A C T
Aims: Administration of estradiol or compounds with estrogenic activity to newborn female rats results in irreversible masculinization as well as defeminization in the brain and the animals exhibit altered reproductive behavior as adults. The cellular and molecular mechanism involved in inducing the irreversible changes is largely unknown. In the present study, we have monitored the changes in the expression of selected synaptogenesis related genes in the sexually dimorphic brain regions such as POA, hypothalamus and pituitary following $17 \beta$-estradiol administration to neonatal female rats.

Main methods: Female Wistar rats which were administered 17ß-estradiol on day 2 and 3 after birth were sacrificed 120 days later and the expression levels of genes implicated in synaptogenesis were monitored by semi-quantitative reverse transcription PCR. Since estradiol induced up-regulation of COX-2 in POA is a marker for estradiol induced masculinization as well as defeminization, in the present study only animals in which the increase in expression of COX-2 gene was observed in POA were included in the study.

Key findings: Down-regulation of genes such as NMDA-2B, NETRIN-1, BDNF, MT-5 MMP and TNF- $\alpha$ was observed in the pre-optic area of neonatally $\mathrm{E} 2$ treated female rat brain but not in hypothalamus and pituitary compared to the vehicle- treated controls as assessed by RT-PCR and Western blot analysis.

Significance: Our results suggest a possibility that down-regulation of genes associated with synaptogenesis in POA, may be resulting in disruption of the cyclical regulation of hormone secretion by pituitary the consequence of which could be infertility and altered reproductive behavior.
\end{abstract}

(c) 2015 Elsevier Inc. All rights reserved.

\section{Introduction}

Exposure to gonadal hormones during the neonatal "critical period" has a long-lasting impact on brain morphology and adult sexual behavior, more so, in terms of reproductive memory [3] one aspect of which is cyclical production of hormones during estrus cycle in rodents or during menstrual cycle in the case of human female. Most of the brain regions have both the aromatase cytochrome $\mathrm{P} 450$, which aromatizes testosterone into estradiol [21] and high levels of estrogen receptors. The most important of all the sexually dimorphic regions of the brain is the SDN-POA (Sexually Dimorphic Nucleus of the Pre-Optic Area), the role of which has been well-established in reproductive physiology and behavior. High incidence of apoptosis was observed in the SDN-POA of female rats of PND 7-10 but not in male rats [8]. This sex difference is, in

\footnotetext{
* Corresponding author at: Department of Biochemistry, Indian Institute of Science, Bangalore 560012, India.

E-mail addresses: ajrao@biochem.iisc.ernet.in, ajrao2006@gmail.com, ajrao_2000@yahoo.com (A.J. Rao).
}

part, responsible for the masculinization of the neonatal brain. Female rats administered gonadal steroid hormones neonatally, have been documented to be infertile and lacking in sexual receptivity in the adulthood [4]. Exposure of newborn female rat to $17-\beta$ estradiol masculinizes the POA and sexual behavior and estradiol-induced masculinization has been known to be brought about by the actions of the prostaglandin E2 (PGE2) in the POA [2]. The mRNA and protein levels of COX-2 (Cyclooxygenase 2), one of the important enzymes in the production of PGE2, are higher in the POA of newborn males than in females and administration of estradiol to new born female rats results in an increase in COX-2 level to that observed in males. This induces a two to three fold increase in dendritic spines (i.e. the male pattern) in the POA, and results in the expression of male sexual behavior during adulthood [33].

Estrogen given neo-natally can stimulate diverse biochemical processes and these may have an effect on the organization of synaptic connections in the brain regions that are involved in sex behavior in the adulthood. Since the process of synaptogenesis involves a coordinated expression of a number of factors especially in the neonatal brain, the expression patterns of some of the genes such as BDNF (Brain-derived neurotrophic factor), tumor necrosis factor alpha (TNF $\alpha$ ), Netrin-1, 
Membrane type 5-matrix metalloproteinase (MT5-MMP) and NMethyl-D-aspartate (NMDA) receptor 2B which are important in controlling synapse formation, synaptic transmission, synaptic plasticity and memory function $[9,16,38]$ were monitored. Many of these genes have a bearing on various functions ranging from cyclical secretion of hormones, induction of ovulation, learning, and memory. In view of this, changes in the expression pattern of some of the genes in the POA, hypothalamus and pituitary were examined in female rats which were administered $17 \beta$-estradiol during neonatal period.

\section{Materials and methods}

\subsection{Animals and Treatment}

Pups born to timed pregnant Wistar rats were housed at the Central Animal Facility, Indian Institute of Science, India, under light: dark $(12 / 12 \mathrm{~h})$ cycle at $24{ }^{\circ} \mathrm{C}$ and $50 \%$ average relative humidity. Animals were maintained in accordance with the guidelines issued by Institutional Animal Ethics Committee (IAEC), Indian Institute of Science and the procedures employed in the study have been approved by the IAEC (CAF/Ethics/164/2009). New born female rats were administered $100 \mu \mathrm{g} /$ day of $17 \beta$-estradiol (Sigma, St. Louis, MO, USA) by subcutaneous route on day 2 and 3 after birth and control littermates were administered sesame oil. E2 was first dissolved in 100\% ethanol and then diluted with sesame oil in the ratio of 1:10. The vehicle used was $10 \%$ ethanol and $90 \%$ oil. The volume of injection was $0.05 \mathrm{ml}(100 \mu \mathrm{g}$ of E2) which was administered slowly enough to minimize leakage and the pups were not returned to the mother until the injection site had sealed enough to be leak free (about 15 min post-injection). The rats were allowed to grow normally and were sacrificed around 120 days during metestrus period by excess $\mathrm{CO}_{2}$ using euthanasia chamber and different regions such as POA, hypothalamus and pituitary of the brain were dissected and stored at $-80^{\circ} \mathrm{C}$. In our study the control and neonatally estradiol exposed rats were sacrificed after 120 days during metestrus period, as all E2 treated animals did not exhibit estrus cycle and are arrested at metestrus stage and our preliminary studies of monitoring changes in expression of genes on day 18, 30 and 60 did not reveal any significant changes in POA.

\subsection{Histology}

In this study, we used Nissl staining to examine the histology of preoptic area by light microscopy. Whole rat brain tissues from control and neonatally estradiol treated female rats (on postnatal day 120) were fixed in $4 \%$ paraformaldehyde and $30-\mu \mathrm{m}$ serial, coronal sections were cut by using vibratome (Leica, Wetzlar, Germany) and mounted directly on gelatine coated slides. After air drying the sections the slides were placed in 1:1 alcohol/chloroform overnight and then rehydrated through $100 \%$ and $95 \%$ alcohol to distilled water and then stained with Cresyl Violet acetate - Nissl stain (Sigma) to visualize anatomical structures. The stained slides were washed in distilled water and dehydrated in ascending percent alcohol, soaked in $100 \%$ xylene for $24 \mathrm{~h}$, and coverslipped with DPX mountant (SRL Chemicals, Mumbai, India). Densitometry of Nissl stained signals (Neuronal density counts) was performed using ImageJ (National Institutes of Health, Bethesda, MD).

\subsection{Semi-quantitative Reverse Transcription Polymerase Chain Reaction (RT-PCR)}

Total RNA from the different regions of the brain was extracted using TRI reagent (Sigma) according to manufacturer's instructions. The integrity of the RNA was checked on 1\% MOPS-HCHO agarose gel and the quantity of RNA was estimated spectrophotometrically. Reverse transcription of $1 \mu \mathrm{g}$ of RNA was carried out at $42{ }^{\circ} \mathrm{C}$ with 200 units of MuMLV-reverse transcriptase and $1.5 \mu \mathrm{M}$ random hexamer primers (Thermo Scientific, MA, USA). A simultaneous control without reverse transcriptase was included to verify the absence of non-specific amplification. $1 \mu \mathrm{l}$ of the cDNA was employed for PCR amplification of different genes using highly specific forward and reverse primers (Table 1). Initially, the annealing temperature and number of cycles were optimized and PCR was performed within the linear range of amplification to facilitate quantitation. cDNA amplifications were achieved using an initial heating at $94{ }^{\circ} \mathrm{C}$ for $3 \mathrm{~min}$, followed by 30 cycles of $94{ }^{\circ} \mathrm{C}$ for $1 \mathrm{~min}$, annealing temperature of $60^{\circ} \mathrm{C}$ for $1 \mathrm{~min}$ and final extension at a temperature of $72^{\circ} \mathrm{C}$ for $10 \mathrm{~min}$, on a PCR Thermal Cycler (MJ Research Inc., Watertown, USA). Gel electrophoresis was carried out by taking $30 \mu \mathrm{l}$ of the PCR product on a $1.5 \%$ agarose gel containing $0.5 \mu \mathrm{g} / \mathrm{ml}$ of Ethidium bromide, in $1 \times$ TBE buffer containing $45 \mathrm{mM}$ Tris-borate and 0.5 mM EDTA. The difference in the intensities of the products following electrophoresis was analyzed using Kodak Electrophoresis and Gel Documentation Analysis System (EDAS-120). The expression levels of specific transcripts were inferred upon normalizing their signal intensities to that of glyceraldehyde 3-phosphate-dehydrogenase (GAPDH), which served as an internal control in this semi-quantitative analysis.

\subsection{Western blot analysis}

Protein lysates were prepared from control and E2 treated brain samples using RIPA buffer containing $50 \mathrm{mM}$ Tris, $150 \mathrm{mM} \mathrm{NaCl}, 0.1 \%$ SDS, $0.5 \%$ sodium deoxycholate, $1 \%$ Triton X 100 and protease inhibitor cocktail (Roche Diagnostics Ltd., Mannheim, Germany). Homogenization was carried out using a hand-held teflon-glass homogenizer. The homogenate was centrifuged at $5000 \mathrm{rpm}$ at $4{ }^{\circ} \mathrm{C}$ for $10 \mathrm{~min}$ and the total protein in the supernatant was estimated spectrophotometrically by Lowry's method [17]. From each group, $40 \mu \mathrm{g}$ of protein was electrophoresed on $10 \%$ sodium dodecyl sulfate polyacrylamide gel and Western blotting was carried out as described [11]. The primary antibodies used were anti-ER-alpha (Cell Signaling Technology, Denver, MA, USA, Cat No. \# 8644, dilution- 1:500; MW 66 kDa), anti-ER-beta (Abnova, \# H00002100-M01, dilution-1:500, MW 37 kDa), anti-COX-2 (Cell Signaling Technology, Cat No. \#12,282, dilution-1:500, MW 74 kDa), anti-bdnf (Sigma), anti-FAK (Cell Signaling Technology, Cat No. \#3285, dilution1:500, MW $125 \mathrm{kDa}$ ), anti-TNF-alpha (17 kDa) (a kind gift from Prof. Anjali Karande, Indian Institute of Science, Bangalore), and antiGAPDH (Cell Signaling Technology, \#2118, dilution-1:2000, MW $37 \mathrm{kDa}$ ). Goat anti-rabbit (GE Healthcare, Buckinghamshire, UK - Cat No. \#NA934) and anti-mouse (GE Healthcare, UK - Cat No. NA931) were used as secondary antibodies at the dilution of 1:5000. Signal intensities of various bands were determined by using the Kodak Electrophoresis and Gel Documentation Analysis System (EDAS-120). The expression levels of the protein products of interest were inferred upon normalizing their signal intensities to that of GAPDH. Values so obtained were used to determine mean \pm SEM for a graphical representation.

\subsection{Statistical analysis}

Data in each experiment are presented as mean \pm SEM $(n=5$ per group). Differences between groups were analyzed by two-tailed Student's $t$ test and $\mathrm{p}$ values $<0.05$ were considered statistically significant.

\section{Results}

\subsection{Validation of the model system}

To ascertain that the female rats have responded to the administration of $17 \beta$-estradiol, the expression level of COX-2 in preoptic area, hypothalamus and pituitary from control and E2 treated animals was monitored. The up-regulation of COX-2 in POA region in the E2 treated animals was considered as a positive response to the E2 treatment [2]. Only those animals in which a significant increase in COX-2 expression 
Table 1

List of primers used in the study.

\begin{tabular}{|c|c|c|c|c|c|}
\hline Sl. no. & Gene & Sequence $5^{\prime} \rightarrow 3^{\prime}$ & Length & Annealing temperature $\left({ }^{\circ} \mathrm{C}\right)$ & Size \\
\hline \multirow[t]{2}{*}{1} & ER- $\alpha$ & F-CATCGATAAGAACCGGAGGA & 20 & 60 & $190 \mathrm{bp}$ \\
\hline & ER- $\alpha$ & R-AAGGTTGGCAGCTCTCATGT & 20 & & \\
\hline \multirow[t]{2}{*}{2} & ER- $\beta$ & F-AAAGTAGCCGGAAGCTGACA & 20 & 60 & 218 bp \\
\hline & ER- $\beta$ & R-CTCCAGCAGCAGGTCATACA & 20 & & \\
\hline \multirow[t]{2}{*}{3} & Granulin & F-AAGGCGAGAACCTGTGAGAA & 20 & 60 & $213 \mathrm{bp}$ \\
\hline & Granulin & R-GAAGCCAATGGGACAACAGT & 20 & & \\
\hline \multirow[t]{2}{*}{4} & FAK & F-CGTGAAGCCTTTTCAAGGAG & 20 & 60 & $225 \mathrm{bp}$ \\
\hline & FAK & R-TCCATCCTCATCCGTTCTTC & 20 & & \\
\hline \multirow[t]{2}{*}{5} & COX-2 & F-AAAGCCTCGTCCAGATGCTA & 20 & 60 & 249 bp \\
\hline & COX-2 & R-ATGGTGGCTGTCTTGGTAGG & 20 & & \\
\hline \multirow[t]{3}{*}{6} & Paxillin & F-GGGACGAGCAATCATTTTGT & 20 & 60 & $207 \mathrm{bp}$ \\
\hline & Paxillin & R-TCCAAGAGGCTCTCCACACT & 20 & & \\
\hline & GAPDH & F-CTCATGACCACAGTCCATGC & 20 & & \\
\hline \multirow[t]{2}{*}{7} & GAPDH & R-TTCAGCTCTGGGATGACCTT & 20 & 60 & $216 \mathrm{bp}$ \\
\hline & NMDA 2B & F-CCAAGAGGAGGAAACAGCAG & 20 & & \\
\hline \multirow[t]{2}{*}{8} & NMDA 2B & R-TGAGGCGAGTTCTCCTTTGT & 20 & 60 & 202 bp \\
\hline & Netrin 1 & F-CCTACTGCAAGGCTTCCAAA & 20 & & \\
\hline \multirow[t]{2}{*}{9} & Netrin 1 & R-CGAACGGATCCACAAACTCT & 20 & 60 & $197 \mathrm{bp}$ \\
\hline & MT5-MMP & F-CGGAACTCCTGTAGGCTCTG & 20 & & \\
\hline \multirow[t]{2}{*}{10} & MT5-MMP & R-GAGGGACCAGGAAACTAGGG & 20 & 60 & 202 bp \\
\hline & BDNF & F-GGTTCGAGAGGTCTGACGAC & 20 & & \\
\hline \multirow[t]{2}{*}{11} & BDNF & R-CGGCATCCAGGTAATTTTTG & 20 & 60 & $211 \mathrm{bp}$ \\
\hline & $\mathrm{TNF} \alpha$ & F-ATGTGGAACTGGCAGAGGAG & 20 & & \\
\hline 12 & TNF $\alpha$ & R-GGCCATGGAACTGATGAGAG & 20 & 60 & $200 \mathrm{bp}$ \\
\hline
\end{tabular}

was observed following E2 administration were used for further studies which consisted of isolating RNA and protein. It is evident from results presented in Fig. 1A \&B that following neonatal administration of E2, there was a significant increase in the expression of mRNA as well as protein levels of COX-2 in POA of neonatally E2 treated animals compared to the controls. It can also be seen from the results presented in Fig. 1C, Nissl staining of SDN-POA showed significant increase in neuronal cell density in $\mathrm{E} 2$ treated group thus validating the model as E2 treatment prevents apoptosis normally seen in the female rats post-natally.

\subsection{Expression pattern of selected genes involved in brain sex differentiation}

The level of expression of ER- $\alpha$, ER- $\beta$, FAK, Paxillin and Granulin in POA, hypothalamus and pituitary of control and E2 treated female rats assessed by semi-quantitative RT-PCR are presented in (Fig. 2, top panel). It was observed that the expression level of ER- $\alpha$ decreased significantly in the POA and hypothalamus. There was a significant decrease in level of expression of ER- $\beta$ in the hypothalamus and also in the pituitary. FAK expression was significantly decreased in all three regions of the brain ( $\mathrm{POA}$, hypothalamus and pituitary). The expression of
A

$\underline{\text { RT-PCR analysis }}$
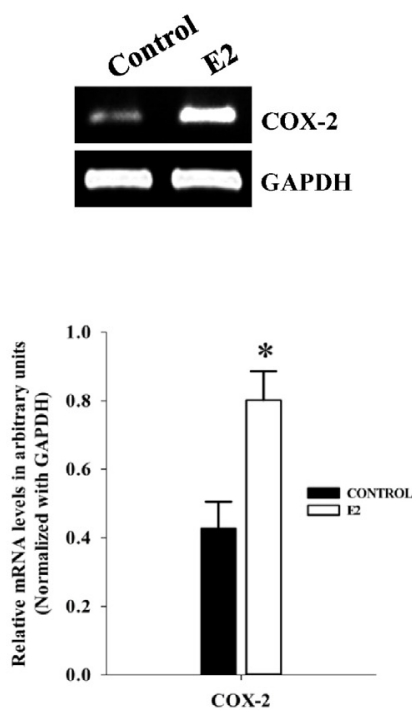

B

Western Blot analysis
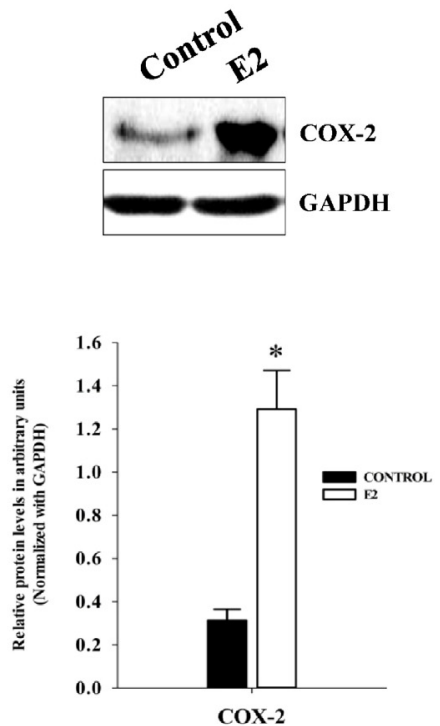

C
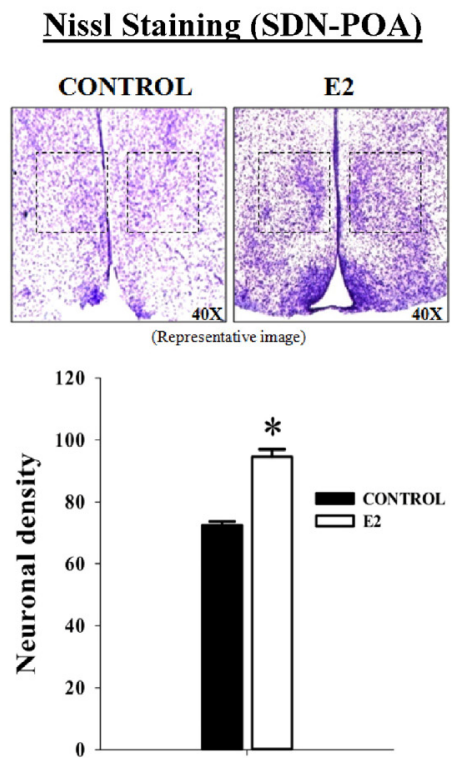

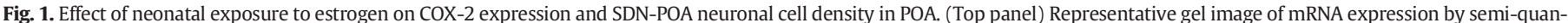

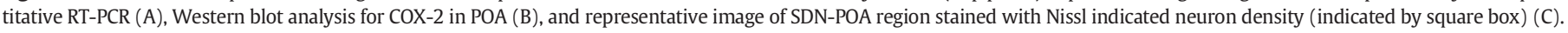

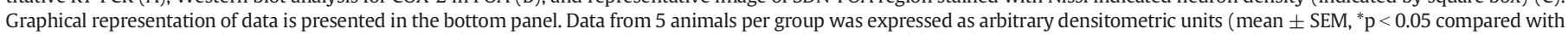
control) for RT-PCR, Western blot and histology. 

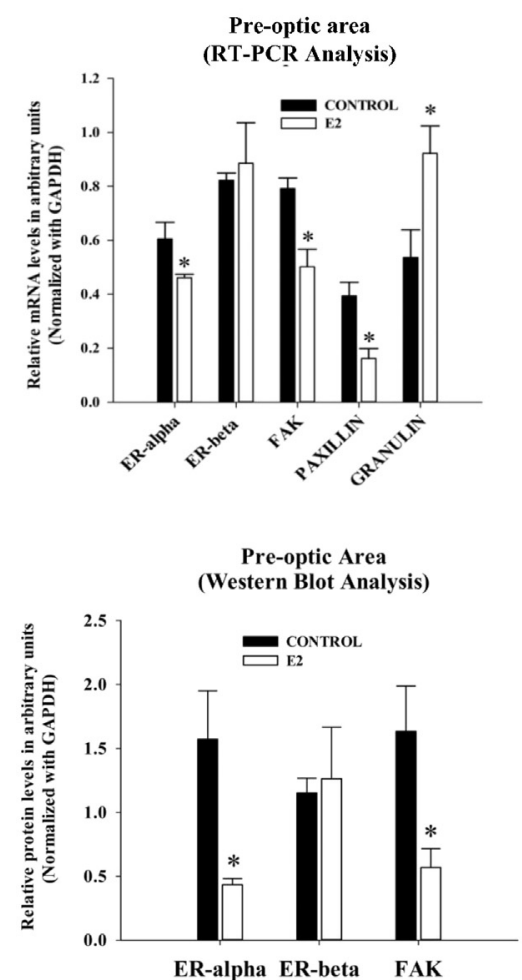

B
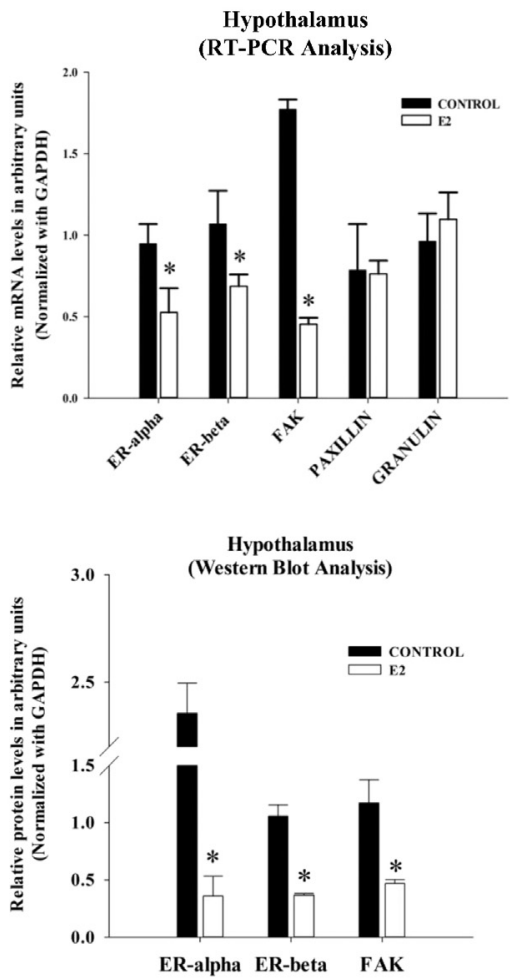

C
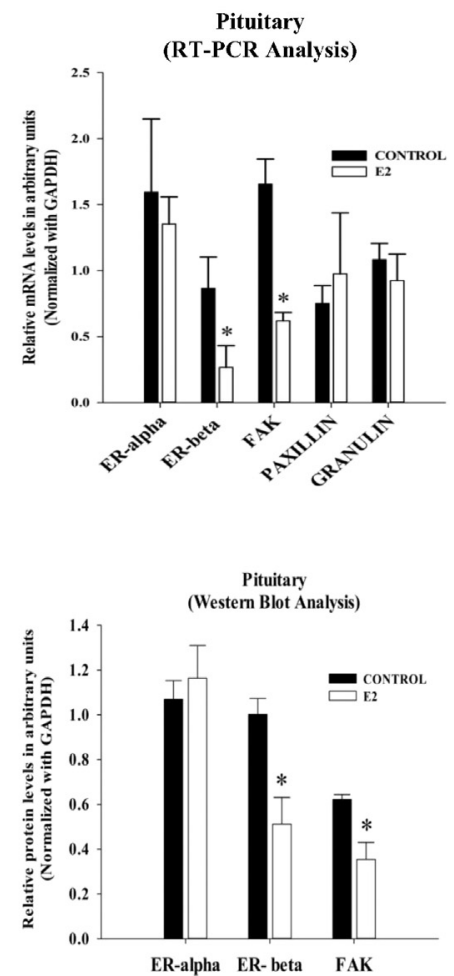

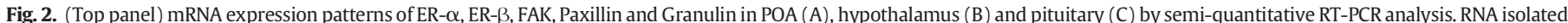

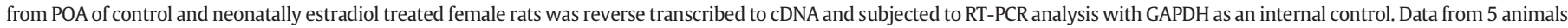

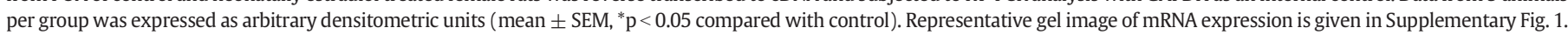

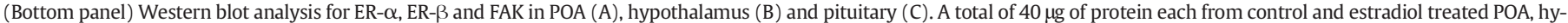

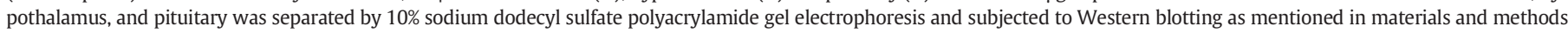

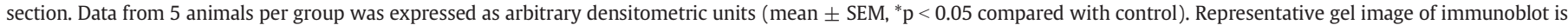
given in Supplementary Fig. 2.

ER- $\alpha$, ER- $\beta$, and FAK was further confirmed by Western blot analysis (Fig. 2, bottom panel). There was no significant difference in the expression levels of Paxillin in all three regions observed. However, a significant up-regulation of Granulin was seen in the POA, but not in hypothalamus or pituitary.

\subsection{Expression pattern of selected genes involved in synaptogenesis}

Synaptogenesis is one of the key processes by which sex differences are established in the brain. Out of the 3 regions investigated, following E2 treatment, a significant down-regulation of genes known to be involved in synaptogenesis was observed only in POA (Fig. 3) suggesting that POA is an important region for regulation of brain sex differentiation. As can be seen from results represented in Fig. 3 (top panel), there is a significant decrease in the expression of mRNA of genes known to regulate synaptogenesis such as NMDA2B, Netrin-1, MT5MMP, BDNF and TNF- $\alpha$ in the POA region. The decrease in BDNF and TNF- $\alpha$ was further confirmed by Western blot analysis (Fig. 3, bottom panel). It is also interesting to note that there is no significant change in the expression pattern of these genes either in the hypothalamus or in the pituitary region.

\section{Discussion}

Experimental studies on animals demonstrated that the exposure of compounds with estrogenic activity to newborn female animals during critical window period results in irreversible alterations in female gonadal function and sexual behavior in the adulthood and the brain sex of a female are converted to that of a male type $[20,29,37]$. In the present study, our observation clearly establishes that neonatal exposure of female rats to high levels of estradiol results in changes in the expression of some of the key genes in POA, hypothalamus, and pituitary which are summarized in Table 2, as assessed both by RTPCR and Western blot analysis. It needs to be emphasized that some of the conclusions drawn on the significance of changes seen in the level of expression of some of the transcripts are based entirely on semi-quantitative RTPCR and Western blot analysis (both of which highly reproducible) of some of the products of genes analyzed. Although validation of the changes in the expression by real-time PCR analysis as well as Western blot analysis for all the transcripts will provide additional support to the conclusions drawn in the present study it could not be carried out due to the lack of access to the highly specific antibodies. In spite of the limitation mentioned above, it is possible to suggest based on the results obtained that these changes perhaps are responsible for irreversible changes seen in lack of cyclical production of hormones following neonatal administration of $17 \beta$-estradiol which ultimately manifests in masculinization or defeminization in the female rat brain. It needs to be emphasized that tissue samples were collected and used in this study only from animals in which there was a significant increase in COX-2 in POA, a parameter considered for the efficacy of estrogen action. It is known that the area occupied by SDN in the POA is equal in both male and female initially, and post-natally there is a drastic decrease in SDN by the process of apoptosis of the neurons in the females and this is prevented by estradiol administration [20]. As can be seen from the current results that there is an increase in the neurons in the SDN-POA of E2 treated female rats compared to POA of intact female rats. This observation of increase in SDN-POA volume confirms evidence for masculinization and defeminization in female rats and this finding is 
A

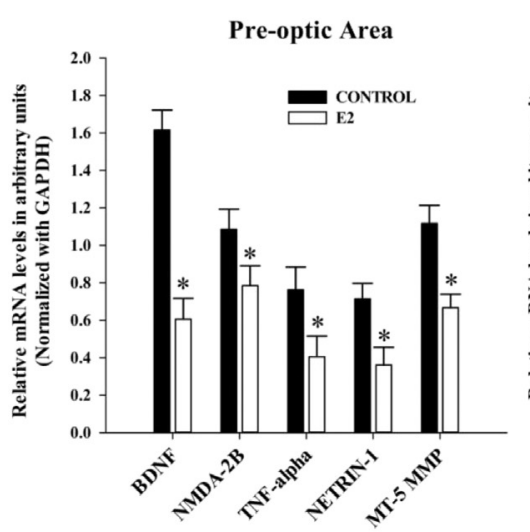

B

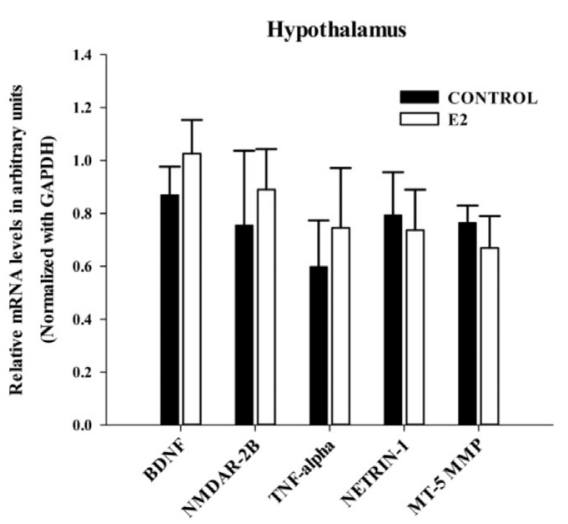

Preoptic area

(Western Blot Analysis)

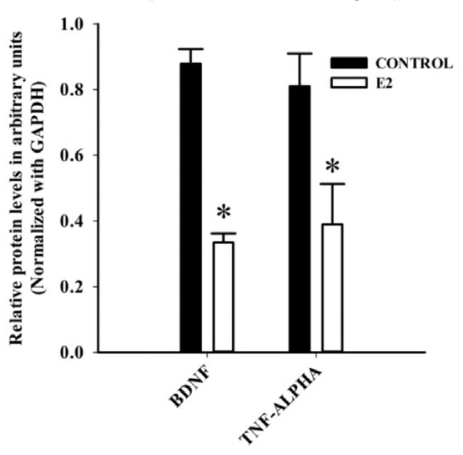

C

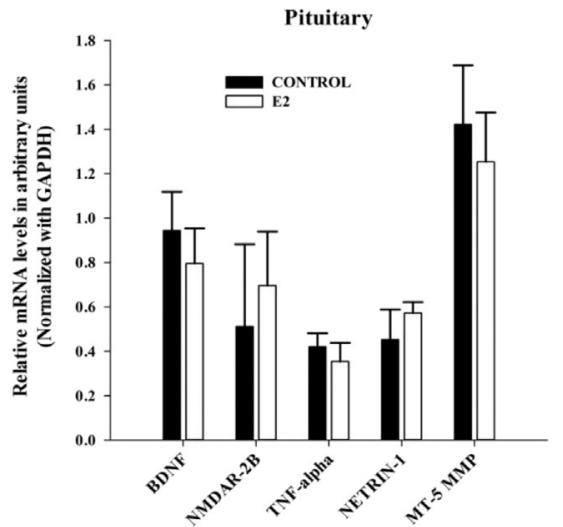

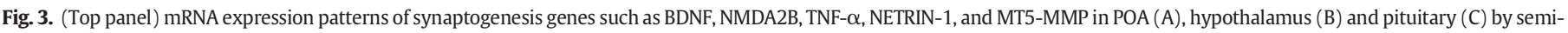

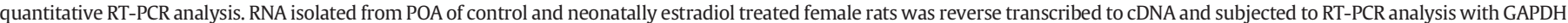

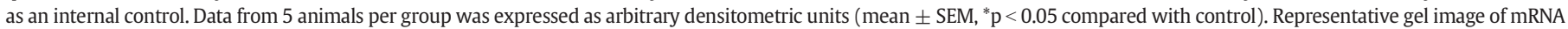

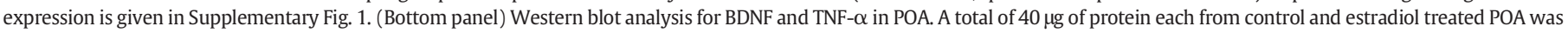

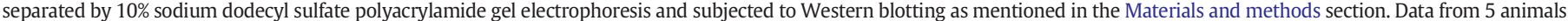
per group was expressed as arbitrary densitometric units (mean \pm SEM, ${ }^{*} \mathrm{p}<0.05$ compared with control). Representative gel image of immunoblot is given in Supplementary Fig. 2.

consistent with observations by others [10,28]. Estrogen sensitivity is critical for maternal behavior and its action is predominantly mediated by the two nuclear estrogen receptor subtypes (ER- $\alpha$ and ER- $\beta$ ) and they are expressed in a sex-specific manner $[25,28]$. ER- $\alpha$ has been shown to be involved in female maternal care and regulating anxiety and fear [13,31]. In the present study, we observed down-regulation of ER- $\alpha$ in POA of estradiol administered female rat which indicates that E2 induces important irreversible changes in terms of compromised feminine behavior as well as maternal care and also establishes defeminization during the adult stage. ER- $\beta$ is important in inducing
LH surge by estrogen, typical of female estrous cycle and downregulation of ER- $\beta$ has been implicated in estrogen induced masculinization of rat hypothalamus [26,27]. Our observation in the current study suggests that the down-regulation of ER- $\beta$ in the hypothalamus, the main region of the brain known to be involved in initiating cyclicity, which involves initiation of secretion of the needed hormones at selected intervals by the pituitary, suggests impairment of the ovulatory process in the E2 treated rats, thereby rendering them infertile. Focal adhesion kinase and Paxillin are important regulators of neurite growth by controlling interaction with the extra cellular matrix via integrins

Table 2

Summary of results obtained.

\begin{tabular}{|c|c|c|c|c|}
\hline & & \multicolumn{3}{|l|}{ Brain regions } \\
\hline & & Preoptic area & Hypothalamus & Pituitary \\
\hline \multirow{12}{*}{ Gene of interest } & Neuronal density & $\uparrow$ (Nissl staining) & $\mathrm{N} / \mathrm{M}$ & $\mathrm{N} / \mathrm{M}$ \\
\hline & ER- $\alpha$ & $\downarrow$ (mRNA \& protein) & $\downarrow$ (mRNA \& protein) & $\leftrightarrow$ \\
\hline & ER- $\beta$ & $\leftrightarrow$ & $\downarrow$ (mRNA \& protein) & $\downarrow$ (mRNA) \\
\hline & $\mathrm{COX}-2$ & $\uparrow($ mRNA \& protein) & $\mathrm{N} / \mathrm{M}$ & $\mathrm{N} / \mathrm{M}$ \\
\hline & FAK & $\downarrow$ (mRNA \& protein) & $\downarrow$ (mRNA \& protein) & $\downarrow$ (mRNA) \\
\hline & Paxillin & $\downarrow$ & $\leftrightarrow$ & $\leftrightarrow$ \\
\hline & Granulin & $\uparrow$ & $\leftrightarrow$ & $\leftrightarrow$ \\
\hline & BDNF & $\downarrow$ (mRNA \& Protein) & $\leftrightarrow$ & $\leftrightarrow$ \\
\hline & NMDA-2B & $\downarrow(\mathrm{mRNA})$ & $\leftrightarrow$ & $\leftrightarrow$ \\
\hline & TNF- $\alpha$ & $\downarrow$ (mRNA \& Protein) & $\leftrightarrow$ & $\leftrightarrow$ \\
\hline & Netrin-1 & $\downarrow(\mathrm{mRNA})$ & $\leftrightarrow$ & $\leftrightarrow$ \\
\hline & MT5-MMP & $\downarrow$ (mRNA) & $\leftrightarrow$ & $\leftrightarrow$ \\
\hline
\end{tabular}

$\uparrow=$ increase; $\downarrow=$ decrease; N/M = not measured; $\leftrightarrow=$ no significant change in mRNA level. 
[19]. FAK is generally high in the hypothalamus of neonatal female [35], and the decrease following estrogen treatment as is evident from the present results indicated that neonatal estradiol establishes irreversible masculinization and defeminization of the female rat brain. Granulin, a cytotropic factor was found to be increased in hypothalamus of male rats after neonatal estradiol treatment [36] but the significance of its increase in expression in POA in our study currently is not known.

Although estradiol has been shown to masculinize and defeminize the female rat brain, as adults, there was no conclusive evidence available to suggest that it is due to the changes in expression of genes known to be involved in brain sex differentiation and synaptogenesis following neonatal exposure to estradiol. The results of the present study indicate that neonatal estradiol exposure significantly downregulates synaptogenesis related genes such as NMDAR 2B, NETRIN-1, BDNF, MT5-MMP and TNF- $\alpha$ in female rat POA compared to control rats as seen during adulthood. One of the differentially expressed gene, namely, NMDA2B is a subunit of NMDA receptor and NMDA receptors are known to have an important role in learning and memory, as well as the formation of excitatory synapses and the synaptic transmission [1]. The other down-regulated genes, Netrin-1 is a novel regulator of synaptogenesis and synaptic function [30,34], MT5-MMP an enzyme known to have a function in synapse remodeling [22] and Tumor necrosis factor- $\alpha$ (TNF- $\alpha)[24,30]$, have been identified as neurotrophic factors which regulate synaptogenesis, synaptic transmission, and plasticity. Similarly the expression of BDNF which decreased in POA in the present study has also been shown to induce growth, differentiation and survival of neurons and synapses during brain development $[5,15]$, as well as in adulthood $[7,18]$. Considering the fact that a significant down-regulation of several important genes involved in sex differentiation and synaptogenesis genes is seen only in the POA and not in any of the other regions analyzed suggests, that POA is the most important region in controlling the reproductive behavior. It is pertinent to suggest that there appears to be a link between neurotropic system and estrogen and the region specific mechanism of estradiol action. This is precisely coordinated to achieve the specific goal of sexual differentiation of the female brain and also the concurrent process of masculinization and defeminization in the adult female rats.

Considering estradiol induced androgenization of female rat brain and changes in synaptogenesis related genes in the present study, an important question that still remains to be answered is whether prenatal steroid masculinize the human brain. In non-human primate, it has been speculated that the sex differentiation of the brain is influenced by androgen when female monkeys exposed to androgens early in development are masculinized with respect to sexual behavior, rough play, grooming and some learning abilities [6]. Although there are no definitive evidences for involvement of estrogen in human sexual brain differentiation, there are some from the "naturally occurring experiments". In the case of congenital adrenal hyperplasia (CAH), in which female fetus is exposed to elevated androgen levels, it is believed that there is some degree of masculinization of the brain consequent to fetal exposure to androgens [12]. Studies in the rat SDN-POA inspired the examination of human POA where a nucleus was found that was smaller in women than in men and smaller in gay men than in straight men [14,23]. A similar difference was seen in sheep, POA is smaller in male sheep than prefer to mount other rams than in males that prefer to mount ewes [32]. These observations do suggest that the brain is important for determining sexual orientation and thus this study is relevant to human situation. The animal data tend to substantiate effects of early exposure to estrogen on hypothalamo-pituitary-gonadal (HPG) axis with alterations of sexual maturation and reproductive functions in female rodents. It has always been controversial to extrapolate from data that are obtained in laboratory animals in which the dose and time of chemical exposure do not always represent conditions that humans face in their habitat during a life time. However, we cannot ignore the increasing evidence coming from these studies when human populations are exposed to the same chemicals during developmental stages.

\section{Conclusion}

Exposure of neonatal female rats to high levels of estrogen or compounds with estrogenic activity results in masculinisation and disturbance in female sexual receptive behavior in the later life. The results of our study show a significant down-regulation of genes associated with synaptogenesis in POA, the consequence of which might be decreased learning and memory because of synaptic structural alteration, ultimately leading to loss of reproductive memory and thereby decreased or diminished female sexual behavior observed in accidental exposure to high levels of estradiol during critical period.

Supplementary data to this article can be found online at http://dx. doi.org/10.1016/j.lfs.2015.09.013.

\section{Acknowledgments}

The author A.J. Rao would like to thank the Indian National Science Academy for the award of INSA Senior Scientist Fellowship and Department of Science and Technology (DST-Government of India) for financial assistance (Grant No. SR/SO/HS-0016/2012) provided during the course of the work. Also the author A.J. Rao would like to thank, Department of Biochemistry, Indian Institute of Science, Bangalore, India.

\section{References}

[1] M.M. Adams, S.E. Fink, W.G. Janssen, R.A. Shah, J.H. Morrison, Estrogen modulates synaptic N-methyl-D-aspartate receptor subunit distribution in the aged hippocampus, J. Comp. Neurol. 474 (2004) 419-426.

[2] S.K. Amateau, M.M. McCarthy, Induction of PGE2 by estradiol mediates developmental masculinization of sex behavior, Nat. Neurosci. 7 (2004) 643-650.

[3] A.P. Arnold, S.M. Breedlove, Organizational and activational effects of sex steroids on brain and behavior: a reanalysis, Horm. Behav. 19 (1985) 469-498.

[4] C.A. Barraclough, Production of anovulatory, sterile rats by single injections of testosterone propionate 1, Endocrinology 68 (1961) 62-67.

[5] M. Bothwell, Functional interactions of neurotrophins and neurotrophin receptors, Annu. Rev. Neurosci. 18 (1995) 223-253.

[6] C.C. Cohen-Bendahan, C. van de Beek, S.A. Berenbaum, Prenatal sex hormone effects on child and adult sex-typed behavior: methods and findings, Neurosci. Biobehav. Rev. 29 (2005) 353-384.

[7] J.C. Conover, G.D. Yancopoulos, Neurotrophin regulation of the developing nervous system: analyses of knockout mice, Rev. Neurosci. 8 (1997) 13-28.

[8] E.C. Davis, P. Popper, R.A. Gorski, The role of apoptosis in sexual differentiation of the rat sexually dimorphic nucleus of the preoptic area, Brain Res. 734 (1996) 10-18.

[9] I.M. Ethell, D.W. Ethell, Matrix metalloproteinases in brain development and remodeling: synaptic functions and targets, J. Neurosci. Res. 85 (2007) 2813-2823.

[10] R.A. Gorski, J.H. Gordon, J.E. Shryne, A.M. Southam, Evidence for a morphological sex difference within the medial preoptic area of the rat brain, Brain Res. 148 (1978) 333-346.

[11] V. Govindaraj, R.K. Basavaraju, A.J. Rao, Changes in the expression of DNA double strand break repair genes in primordial follicles from immature and aged rats, Reprod. BioMed. Online (2014).

[12] M. Hines, Prenatal endocrine influences on sexual orientation and on sexually differentiated childhood behavior, Front. Neuroendocrinol. 32 (2011) 170-182.

[13] A.E. Kudwa, V. Michopoulos, J.D. Gatewood, E.F. Rissman, Roles of estrogen receptors alpha and beta in differentiation of mouse sexual behavior, Neuroscience 138 (2006) 921-928.

[14] S. LeVay, A difference in hypothalamic structure between heterosexual and homosexual men, Science 253 (1991) 1034-1037.

[15] G.R. Lewin, Y.-A. Barde, Physiology of the neurotrophins, Annu. Rev. Neurosci. 19 (1996) 289-317.

[16] D. Liu, J. Diorio, J.C. Day, D.D. Francis, M.J. Meaney, Maternal care, hippocampal synaptogenesis and cognitive development in rats, Nat. Neurosci. 3 (2000) 799-806.

[17] O.H. Lowry, N.J. Rosebrough, A.L. Farr, R.J. Randall, Protein measurement with the Folin phenol reagent, J. Biol. Chem. 193 (1951) 265-275.

[18] B. Lu, A. Figurov, Role of neurotrophins in synapse development and plasticity, Rev. Neurosci. 8 (1997) 1-12.

[19] M. McCarthy, How it's made: organisational effects of hormones on the developing brain, J. Neuroendocrinol. 22 (2010) 736-742.

[20] M.M. McCarthy, Estradiol and the developing brain, Physiol. Rev. 88 (2008) 91-134

[21] B.S. McEwen, I. Lieberburg, C. Chaptal, L.C. Krey, Aromatization: important for sexual differentiation of the neonatal rat brain, Horm. Behav. 9 (1977) 249-263.

[22] S. Monea, B.A. Jordan, S. Srivastava, S. DeSouza, E.B. Ziff, Membrane localization of membrane type 5 matrix metalloproteinase by AMPA receptor binding protein and cleavage of cadherins, J. Neurosci. 26 (2006) 2300-2312.

[23] J.A. Morris, C.L. Jordan, S.M. Breedlove, Sexual differentiation of the vertebrate nervous system, Nat. Neurosci. 7 (2004) 1034-1039.

[24] M.A. Muñoz-Fernández, M. Fresno, The role of tumour necrosis factor, interleukin 6, interferon- $\gamma$ and inducible nitric oxide synthase in the development and pathology of the nervous system, Prog. Neurobiol. 56 (1998) 307-340. 
[25] B.M. Nugent, J.M. Schwarz, M.M. McCarthy, Hormonally mediated epigenetic changes to steroid receptors in the developing brain: implications for sexual differentiation, Horm. Behav. 59 (2011) 338-344.

[26] C. Orikasa, Y. Kondo, S. Hayashi, B.S. McEwen, Y. Sakuma, Sexually dimorphic expression of estrogen receptor beta in the anteroventral periventricular nucleus of the rat preoptic area: implication in luteinizing hormone surge, Proc. Natl. Acad. Sci. U. S. A. 99 (2002) 3306-3311.

[27] C. Orikasa, Y. Sakuma, Sex and region-specific regulation of oestrogen receptor $\beta$ in the rat hypothalamus, J. Neuroendocrinol. 16 (2004) 964-969.

[28] A.V. Patchev, F. Götz, W. Rohde, Differential role of estrogen receptor isoforms in sex-specific brain organization, FASEB J. 18 (2004) 1568-1570.

[29] H.B. Patisaul, E.K. Polston, Influence of endocrine active compounds on the developing rodent brain, Brain Res. Rev. 57 (2008) 352-362.

[30] V.Y. Poon, S. Choi, M. Park, Growth factors in synaptic function, Front. Synaptic Neurosci. 5 (2013)

[31] A.C. Ribeiro, S. Musatov, A. Shteyler, S. Simanduyev, I. Arrieta-Cruz, S. Ogawa, et al., siRNA silencing of estrogen receptor-alpha expression specifically in medial preoptic area neurons abolishes maternal care in female mice, Proc. Natl. Acad. Sci. U. S. A. 109 (2012) 16324-16329.
[32] C.E. Roselli, K. Larkin, J.A. Resko, J.N. Stellflug, F. Stormshak, The volume of a sexually dimorphic nucleus in the ovine medial preoptic area/anterior hypothalamus varies with sexual partner preference, Endocrinology 145 (2004) 478-483.

[33] J.M. Schwarz, M.M. McCarthy, Cellular mechanisms of estradiol-mediated masculinization of the brain, J. Steroid Biochem. Mol. Biol. 109 (2008) 300-306.

[34] K. Shen, C.W. Cowan, Guidance molecules in synapse formation and plasticity, Cold Spring Harb. Perspect. Biol. 2 (2010) a001842.

[35] D.B. Speert, A.T. Konkle, S.L. Zup, J.M. Schwarz C. Shiroor, M.E Taylor, et al., Focal adhesion kinase and paxillin: novel regulators of brain sexual differentiation? Endocrinology 148 (2007) 3391-3401.

[36] M. Suzuki, M. Nishiahara, Granulin precursor gene: a sex steroid-inducible gene involved in sexual differentiation of the rat brain, Mol. Genet. Metab. 75 (2002) 31-37.

[37] C.L. Wright, J.S. Schwarz, S.L. Dean, M.M. McCarthy, Cellular mechanisms of estradiol-mediated sexual differentiation of the brain, Trends Endocrinol. Metab. 21 (2010) 553-561.

[38] K. Yamada, T. Nabeshima, Brain-derived neurotrophic factor/TrkB signaling in memory processes, J. Pharmacol. Sci. 91 (2003) 267-270. 